Now, John Wiley and co-workers at the University of New Orleans and Louisiana State University in the USA have used a combination of two well-known techniques to produce patterned arrays of either silver, gold or nickel nanowires. By using a standard photolithographic procedure, a particular pattern - as defined by the photo-mask - can be created on top of a porous alumina template, thereby sealing off the pores it covers. The surface is then coated with a thin layer of silver or gold, which serves as an electrode for the electrochemical deposition of metal - from the other side - into the pores that were not initially sealed. A sodium hydroxide wash removes the alumina template, leaving behind a pattern of freestanding metallic nanowires.

A wide range of patterns can be generated using different masks, and individual nanowire dimensions can be controlled by varying the pore size of the template and the duration of the deposition process. Although Wiley and co-workers have focused on metal nanowire patterns in this study, they suggest that the process could be applied to other materials such as oxides and semiconductors.

\section{MASS SPECTROMETRY}

\section{Weighing up surfaces}
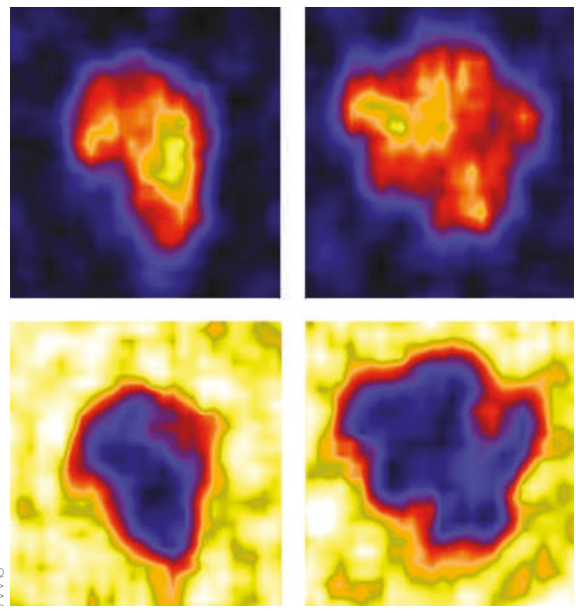

Science 313, 1948-1951 (2006)

Mass spectrometry is an analytical technique that can be used to weigh molecules. Although many variations exist, the basic principles are the same; the sample of interest is ionized and the ions are separated, according to their mass-to-charge ratio, and counted. Now, researchers in the USA have shown how mass spectrometry can be used to map out the distribution of two different lipids in a membrane.

Steven Boxer and co-workers from

Stanford University, the University of California, Davis, and Lawrence Livermore National Laboratory look at bilayers in which two different isotopically labelled lipids separate into domains. As a focused beam of caesium ions scans across the surface of a freezedried membrane, the lipid molecules fragment to form ions. These 'secondary' ions - which retain the isotopic signature of their parent lipids - are then detected and give a spatially resolved picture of the membrane.

Individual membrane components can be resolved at the molecular level with electron microscopy, and optical imaging probes much larger length scales. Boxer's technique falls in between, however, and can image the lateral organization of lipids within a membrane with $100 \mathrm{~nm}$ resolution as well as providing chemical composition data.

\section{SPINTRONICS \\ Mix and match}

Phys. Rev. Lett. 97, 146101 (2006)

Thin films of MnGa and GaAs doped with $\mathrm{Mn}$ atoms are being widely studied for fabricating spintronic devices. Such materials are usually deposited on GaAs substrates by molecular beam epitaxy - a process where evaporated atoms condense on a surface and can react with one another. The growth of MnGa on $\mathrm{GaN}$ substrates has proved to be difficult, however, because Mn and Ga form noncrystalline phases that do not exhibit the sort of magnetic properties necessary for spintronic applications.

Now, Erdong Lu and colleagues of Ohio University and Ohio State University in the USA have successfully deposited crystalline MnGa thin films on GaN substrates. Using a molecular-beam epitaxy system fitted with a nitrogen plasma source, high-quality films can be grown in which the ratio of $\mathrm{Mn}$ to $\mathrm{Ga}$ is carefully controlled. Film growth was monitored by scanning tunnelling microscopy and a high-energy electron diffraction technique.

Significantly, the magnetic moments of the films depend on their composition and structure, which can be varied by altering the flux of either the $\mathrm{Mn}$ or $\mathrm{Ga}$ atoms. This discovery offers an additional degree of freedom in the design and construction of optical spintronic devices operating at short wavelengths.

\section{TOP DOWN BOTTOMUP Viruses beware}

Chemistry and physics join the fight against infectious diseases.

Ralph Tripp, professor of infectious diseases at the University of Georgia in the USA, was looking for a way to detect viruses that was both rapid and sensitive to different strains of the same virus, but did not involve the use of antibodies or costly polymerase chain reaction assays. It was at a nanotechnology meeting organized by his university that he heard about surface-enhanced Raman spectroscopy (SERS), a popular technique for analysing surfaces and identifying chemical compounds, and met colleagues from the physics and chemistry departments. This multidisciplinary collaboration has now demonstrated the potential of SERS for detecting trace levels of viruses such as the respiratory virus that infects children, the elderly and people with compromised immune systems (Nano Lett. doi:10.1021/nl061666f; 2006).

The SERS approach involves illuminating a sample and detecting Raman shifts in the light that is scattered. If the sample is placed on a suitably nanostructured metal surface, the signal is enhanced by orders of magnitude, which improves the sensitivity of the technique.

In the Georgia collaboration, Tripp prepared samples of different viruses, Yiping Zhao fabricated the substrates from silver nanorods, and Richard Dluhy made the measurements. The SERS spectra revealed that each virus had its own 'molecular fingerprint'. Moreover, the different strains could be easily identified by comparing the shifts and relative intensities of the peaks in the spectra.

As a Georgia Research Alliance scholar, Tripp was able to get the project started with funds from his own laboratory, and his colleagues did the same. However, he admits that "it is often difficult to 'learn the language' of different sciences, particularly physics". It is also important to be persistent and to be a good communicator, he adds. "I guess it is a lot like being a salesman."

The definitive versions of these Research Highlights first appeared on the Nature Nanotechnology website, along with other articles that will not appear in print. If citing these articles, please refer to the web version. 